\title{
Zeckenmythen und -fakten im Spiegel der Zeit
}

\author{
Philippe Rafeiner
}

Medizinische Klinik Spital Zofingen

Wenn es um Zecken geht, lernen wir ständig dazu. So steigt das Wissen in Bezug auf die Interaktion und Adaptation zwischen Zecke und Wirt, die die Übertragung von Krankheitserregern möglich machen (Stichwort Glykobiologie). Fortschreitende Klimaveränderungen und molekulare Adaptation werden das Spektrum von übertragenen Erkrankungen und die Inzidenz im Lauf der Zeit weiter vergrössern.

In der Schweiz sind aktuell Borrelien die am häufigsten durch Zecken übertragenen Erreger (mehrere Tausend Arztbesuche pro Jahr). Auf dem Vormarsch sind bekanntlich die FSME (Frühsommer-Meningoenzephalitis) mit derzeit im ersten Halbjahr 2020 rund 200 Fallmeldungen. Aber auch die Tularämie nimmt stetig zu (Meldepflicht seit 2004, Fallzahlen im Vergleich zur FSME rund halb so hoch, eine Erkrankung, der ebenfalls zu rund $60 \%$ als Vektor die Zecke zugrunde liegt [1]. Hingegen sind in der Schweiz Erkrankungen an Rickettsien (Rickettsia helvetica), Anaplasmen, Babesien und Ehrlichien noch selten. Beispiele von Zecken-Kolonisationen bekannter Erreger mit bis dato nur fraglichem Übertragungsrisiko sind Bartonellen und Coxiellen.

Das von Nicoletti und Martinez beschriebene Fallbeispiel einer Tularämie hebt die Bedeutung der klinischen Untersuchung hervor [2]. Die Suche nach dem Eschar: eine kleinfleckige Rötung mit zentraler Nekrotisierung, die Tage bis Wochen persistieren kann. Kannten wir die Eschars bisher v.a. bei Afrika-Rückkehrern (beispielsweise aus dem Krüger-Nationalpark) im Zusammenhang mit Rickettsiosen («rickettsia spotted fever», z.B. Rickettsia africae), so sehen wir in der Schweiz aquirierte Eschars nun zunehmend als Ausdruck einer (ulcero-glandulären Form einer) Tularämie (seltenere Ätiologien von infektiös bedingten Eschars sind auch Anthrax oder Ecthyma gangrenosum z.B. durch Pseudomonas aeruginosa und angioinvasive Mykosen bei Immundefizienz).

Eschars durch Rickettsien und Francisella tularensis, aber auch das durch Borrelia burgdorferi verursachte Ery- thema migrans haben eine wichtige gemeinsame diagnostische und therapeutische Gemeinsamkeit: Die Diagnose und damit auch der Therapieentscheid erfolgen primär rein klinisch, da es bisweilen mehrere Wochen bis zur Serokonversion dauert!

Und der Mythos? Obwohl im englischen Sprachraum der «tick bite» (analog dem «insect bite», also dem Insektenstich) ein stehender Begriff ist (und wohl zunehmend in unserem Sprachraum anglizierend als «Zeckenbiss» Eingang findet), beisst die Zecke nicht, sondern sie sticht. Hierfür stehen der Zecke um das Maul herum angeordnete Werkzeuge (Cheliceren) für einen lanzettartigen Einschnitt in die Haut zur Verfügung, es wird der Stechrüssel (Hypostom) vorgeschoben und dann gesaugt. Dieser Vorgang geht für den Wirt aufgrund der Abgabe eines Lokalanästhetikums völlig indolent vor sich, sodass letztendlich nur etwa die Hälfte der Zeckenstiche überhaupt bemerkt werden.

\section{Bibliografie}

Bundesamt für Gesundheit. Zeckenübertragene Krankheiten Lagebericht Schweiz. Bundesamt für Gesundheit: 2020. https://www.bag.admin.ch/bag/de/home/krankheiten/ ausbrueche-epidemien-pandemien/aktuelle-ausbruecheepidemien/zeckenuebertragene-krankheiten.html; letzter Zugriff. 17.08.2020.

Nicoletti GJ, Martinez AE. Wie lautet Ihre Diagnose? Praxis.2020; 109:957-959.

\section{Dr. med. Philippe Rafeiner}

FMH Innere Medizin und Infektiologie

Leitender Arzt, Stv. Chefarzt

Innere Medizin und Diagnostik

Spital Zofingen AG

Mühlethalstrasse 27

4800 Zofingen

philippe.rafeiner@spitalzofingen.ch 